\title{
Stage IIIA Eyelid Carcinoma AJCC v8
}

National Cancer Institute

\section{Source}

National Cancer Institute. Stage IIIA Eyelid Carcinoma A/CC v8. NCI Thesaurus. Code C140523.

Stage IIIA includes: Any T, N1, M0. N1: Metastasis in a single ipsilateral regional lymph node, measuring $3 \mathrm{~cm}$ or less in greatest dimension. M0: No distant metastasis. (AJCC 8th ed.) 